\title{
1 KPTN is a differentially expressed gene in human metastatic breast cancer, in the brain and in the lymph nodes.
}

Shahan Mamoor, $\mathrm{MS}^{1}$

${ }^{1}$ shahanmamoor@gmail.com

East Islip, NY USA

Metastasis to the brain is a clinical problem in patients with breast cancer ${ }^{1-3}$. We mined published microarray data ${ }^{4,5}$ to compare primary and metastatic tumor transcriptomes for the discovery of genes associated with brain metastasis in humans with metastatic breast cancer. We found that kaptin, encoded by KPTN, was among the genes whose expression was most different in the brain and lymph node metastases of patients with metastatic breast cancer. KPTN mRNA was present at increased quantities in brain metastatic tissues as compared to primary tumors of the breast. Importantly, expression of KPTN in primary tumors was significantly correlated with patient recurrence-free survival in patients with breast cancer. Modulation of KPTN expression may be relevant to the biology by which tumor cells metastasize from the breast to the brain while evading immune clearance in the lymph nodes in humans with metastatic breast cancer. These data are one piece of evidence suggesting a common ancestor or tumor clone for brain and lymph node metastases that originate from the primary tumor, alluding to patterns in developmental origin and migratory pathways through the lymph node in human brain metastatic breast cancer.

Keywords: breast cancer, metastasis, brain metastases, central nervous system metastases, lymph node metastases, kaptin, KPTN, systems biology of breast cancer, targeted therapeutics in breast cancer. 
One report described a $34 \%$ incidence of central nervous system metastases in patients

\section{Methods}

We used datasets GSE10893 ${ }^{4}$ and GSE124648 5 for this global differential gene expression analysis of brain metastatic breast cancer in conjunction with GEO2R. GSE10893 was generated using Agilent-011521 Human 1A Microarray G4110A technology with $n=11$ primary breast tumors and $n=3$ brain metastases from patients with breast cancer; analysis was performed using platform GPL885. GSE124648 was generated using Affymetrix Human Genome U133A array technology with $n=10$ normal breast tissues and $n=44$ lymph node metastases from patients with breast cancer; analysis was performed using platform GPL96. The Benjamini and Hochberg method of $p$-value adjustment was used for ranking of differential expression but raw $p$-values were used to assess statistical significance of global differential expression. Log-transformation of data was auto-detected, and the NCBI generated category of platform annotation was used. A different between primary tumors of the breast and brain metastases in humans with breast cancer using a two-tailed t-test. For Kaplan-Meier survival analysis, we used the Kaplan-Meier plotter online tool ${ }^{7}$ for correlation of KPTN mRNA expression levels in primary tumors of the breast with recurrence-free survival in $n=3951$ breast cancer patients.

\section{$\underline{\text { Results }}$}

We performed global comparative transcriptome analysis of metastatic and primary tumor tissues of patients with metastatic breast, as well as normal tissues of the breast using published microarray data ${ }^{4,5}$ to describe the transcriptional landscape of brain metastasis in human breast cancer in an unbiased fashion and for the discovery of novel therapeutic targets.

\section{KPTN is differentially expressed in the brain metastases of patients with brain metastatic breast cancer.}

Through blind, systems-level analysis of published microarray data ${ }^{4}$, we identified kaptin, encoded by KPTN, as a differentially expressed gene in the brain metastatic tissues of humans with breast cancer (Table 1). When sorting each of the genes expressed in brain 
metastases based on significance of difference as compared to primary tumors of the breast in patients with breast cancer, KPTN ranked 222 out of 17418 total transcripts (Chart 1), equating to 98.7\% differential expression. Differential expression of KPTN in the brain metastases of patients with metastatic breast cancer was statistically significant (Chart $1 ; p=1.03 \mathrm{E}-03$ ).

\section{KPTN is differentially expressed in the lymph node metastases of patients with breast cancer.}

\section{KPTN is expressed at higher levels in the brain metastases of patients with metastatic} breast cancer.

We obtained exact mRNA expression levels for KPTN, in primary tumors of the breast and in brain metastasis of patients with brain metastatic breast cancer to determine direction and statistical significance of change in KPTN expression in brain metastatic tissues. We observed compared to primary tumors of the breast: KPTN was expressed at $0.13 \pm 0.46$ arbitrary units (AU) in primary tumors of the breast, while it was expressed at $1.27 \pm 0.25 \mathrm{AU}$ in brain metastatic tissues (Figure 1). The difference in KPTN mRNA levels between primary tumors of the breast and brain metastatic tissues was statistically significant (Figure 1; $p=0.001657$ ).

\section{KPTN expression is correlated with survival outcomes in human breast cancer.}

We performed Kaplan-Meier survival analysis ${ }^{7}$ in 3951 breast cancer patients in total, to evaluate whether KPTN tumor expression was correlated with survival outcomes in breast cancer. We observed a correlation between primary tumor expression of KPTN and recurrence-free survival (RFS) in patients with breast cancer (Figure 2). Patients whose primary tumors expressed low levels of KPTN possessed median RFS of 44 months, while patients whose tumors expressed high levels of KPTN possessed median RFS of 54 months. This difference in RFS based on KPTN tumor expression in patients with breast cancer trended towards statistical significance (Figure 2, Chart 3; logrank $p$-value: 0.001657; hazard ratio: 0.88 (0.79-0.98)). 
Thus, by mining published microarray data ${ }^{4,5}$ in an unbiased and systematic fashion, we identified kaptin, encoded by KPTN, as among the genes whose expression was most different, transcriptome-wide, in the brain and lymph node metastases of patients with breast cancer, when compared to primary tumors of the breast and to normal breast tissues, respectively; we observed significantly increased expression of KPTN in brain metastases as compared to primary tumors of the breast. Further, we found a correlation between KPTN expression and patient survival outcomes, as recurrence-free survival was greater in patients whose primary tumors expressed higher levels of KPTN as compared to patients whose primary tumors expressed lower levels of KPTN.

\section{Discussion}

We provided evidence here that KPTN is among the genes whose expression is most different in the brain and lymph node metastases of patients with metastatic breast cancer, that KPTN mRNA is present at significantly increased quantity in brain metastatic tissues as compared to primary tumors of the breast, and that primary tumor KPTN expression is significantly correlated with patient survival outcomes in human breast cancer. Evaluation of the effects of genetic depletion of KPTN in mouse models of metastatic breast cancer on metastasis to the central nervous system is merited. Modulation of KPTN expression may be relevant to the processes by which breast cancer cells exit the breast, enter the vasculature and/or lymphatics, reside in the lymph nodes, evade immune clearance, breach the blood-brain barrier and colonize the brain. These data are one piece of evidence that allude to a common cell or cell(s) of origin for brain and lymph node metastases in human metastatic breast cancer. 


\section{References}

1. Lin, N.U., Amiri-Kordestani, L., Palmieri, D., Liewehr, D.J. and Steeg, P.S., 2013. CNS metastases in breast cancer: old challenge, new frontiers.

2. Bendell, J.C., Domchek, S.M., Burstein, H.J., Harris, L., Younger, J., Kuter, I., Bunnell, C., Rue, M., Gelman, R. and Winer, E., 2003. Central nervous system metastases in women who receive trastuzumab-based therapy for metastatic breast carcinoma. Cancer, 97(12), pp.2972-2977.

3. Tsukada, Y., Fouad, A., Pickren, J.W. and Lane, W.W., 1983. Central nervous system metastasis from breast carcinoma autopsy study. Cancer, 52(12), pp.2349-2354.

4. Weigman, V.J., Chao, H.H., Shabalin, A.A., He, X., Parker, J.S., Nordgard, S.H., Grushko, T., Huo, D., Nwachukwu, C., Nobel, A. and Kristensen, V.N., 2012. Basal-like Breast cancer DNA copy number losses identify genes involved in genomic instability, response to therapy, and patient survival. Breast cancer research and treatment, 133(3), pp.865-880.

5. Sinn, B.V., Fu, C., Lau, R., Litton, J., Tsai, T.H., Murthy, R., Tam, A., Andreopoulou, E., Gong, Y., Murthy, R. and Gould, R., 2019. SET ER/PR: a robust 18-gene predictor for sensitivity to endocrine therapy for metastatic breast cancer. NPJ breast cancer, 5(1), pp.1-8.

6. Awada, A., Colomer, R., Inoue, K., Bondarenko, I., Badwe, R.A., Demetriou, G., Lee, S.C., Mehta, A.O., Kim, S.B., Bachelot, T. and Goswami, C., 2016. Neratinib plus paclitaxel vs trastuzumab plus paclitaxel in previously untreated metastatic ERBB2-positive breast cancer: the NEfERT-T randomized clinical trial. JAMA oncology, 2(12), pp.1557-1564.

7. Györffy, B., Lanczky, A., Eklund, A.C., Denkert, C., Budczies, J., Li, Q. and Szallasi, Z., 2010. An online survival analysis tool to rapidly assess the effect of 22,277 genes on breast cancer prognosis using microarray data of 1,809 patients. Breast cancer research and treatment, 123(3), pp.725-731. 


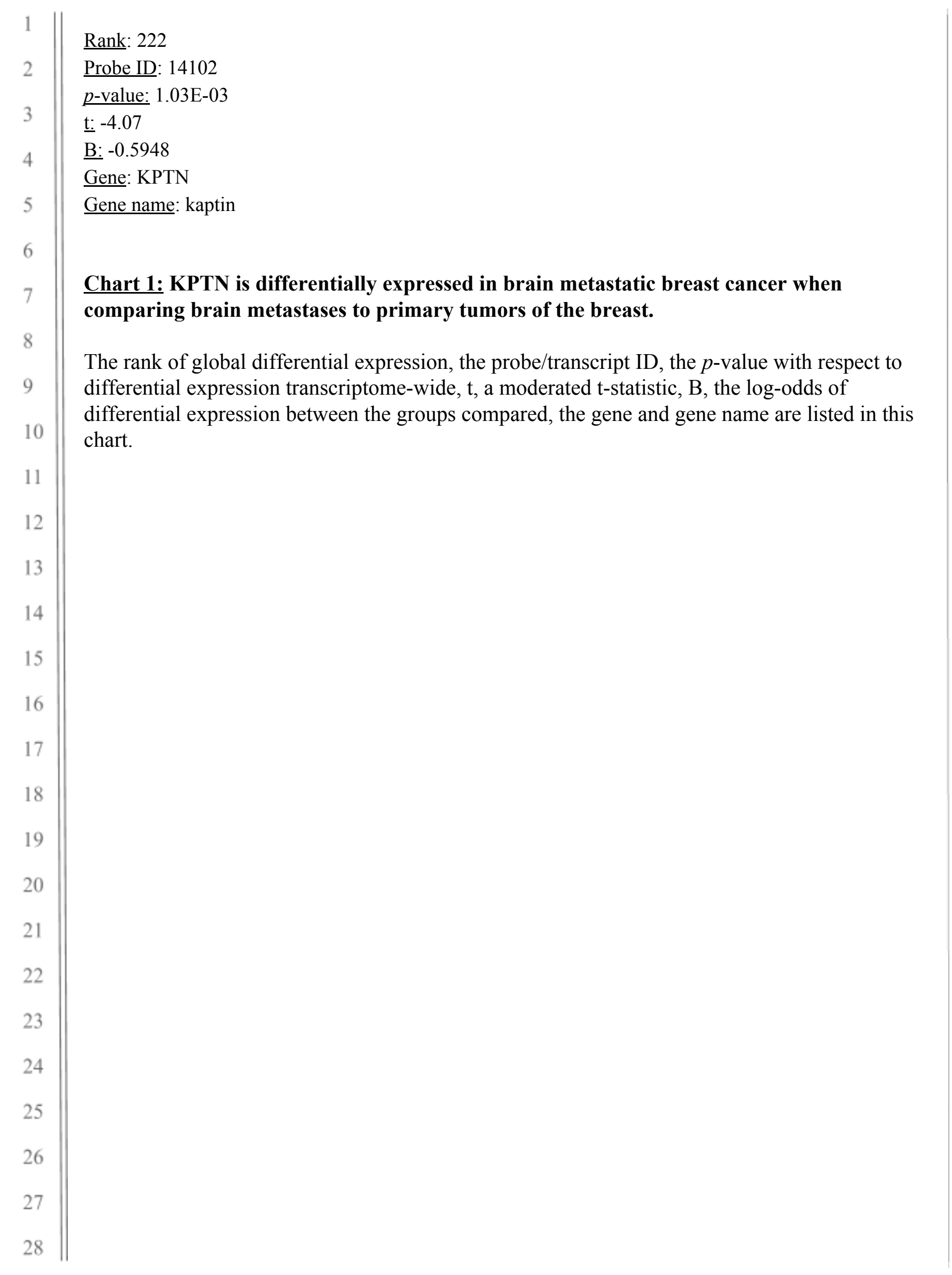




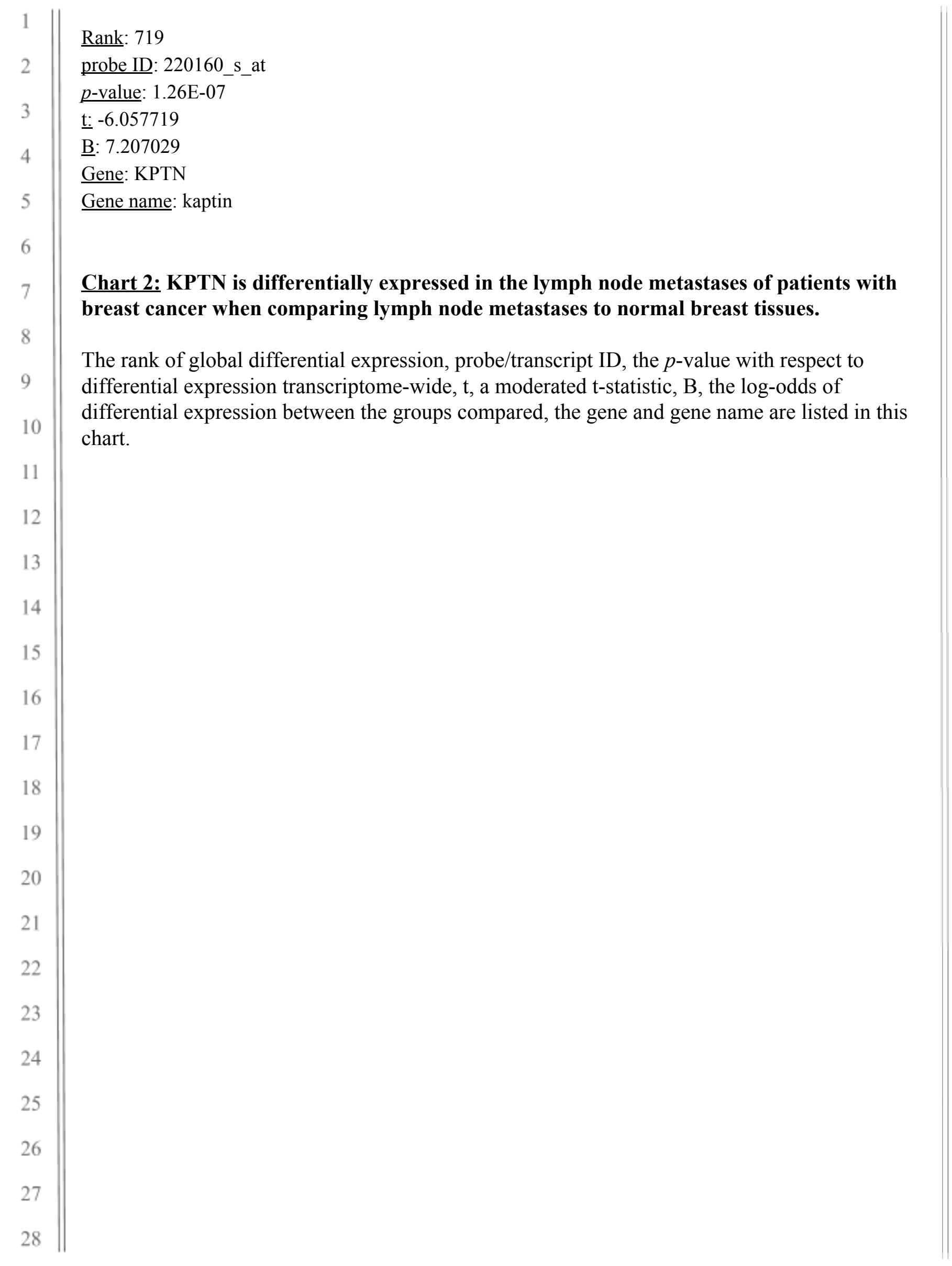


1.5

Figure 1: KPTN is expressed at significantly higher levels in the brain metastases of patients with metastatic breast cancer when compared to primary tumors of the breast.

The mRNA expression level of KPTN in primary tumors of the breast (left) and in brain metastases of women with metastatic breast cancer (right) is graphically depicted; the result of a statistical test evaluating significance of difference in KPTN expression between primary tumors and brain metastases is $p=0.001657$. 


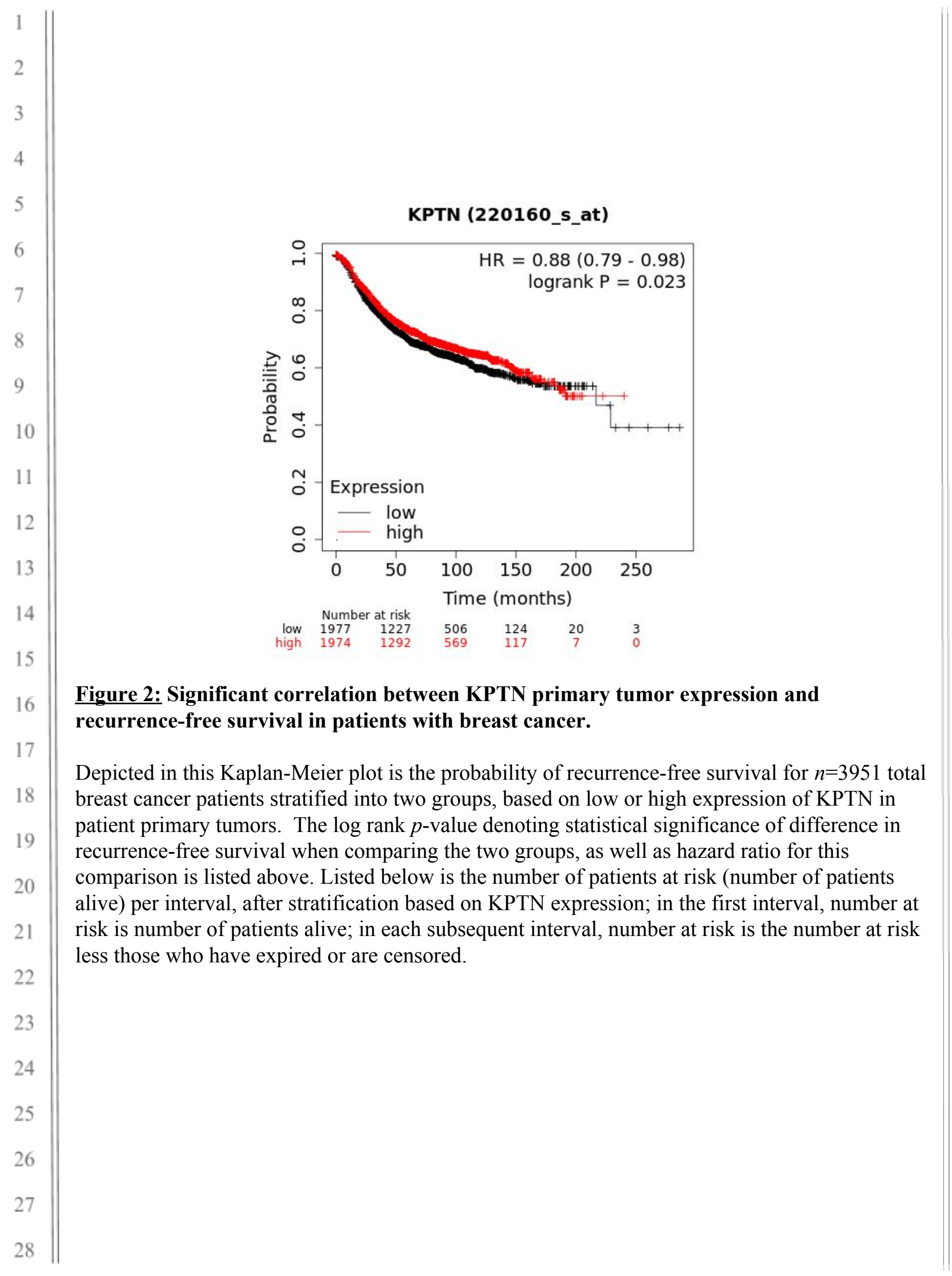




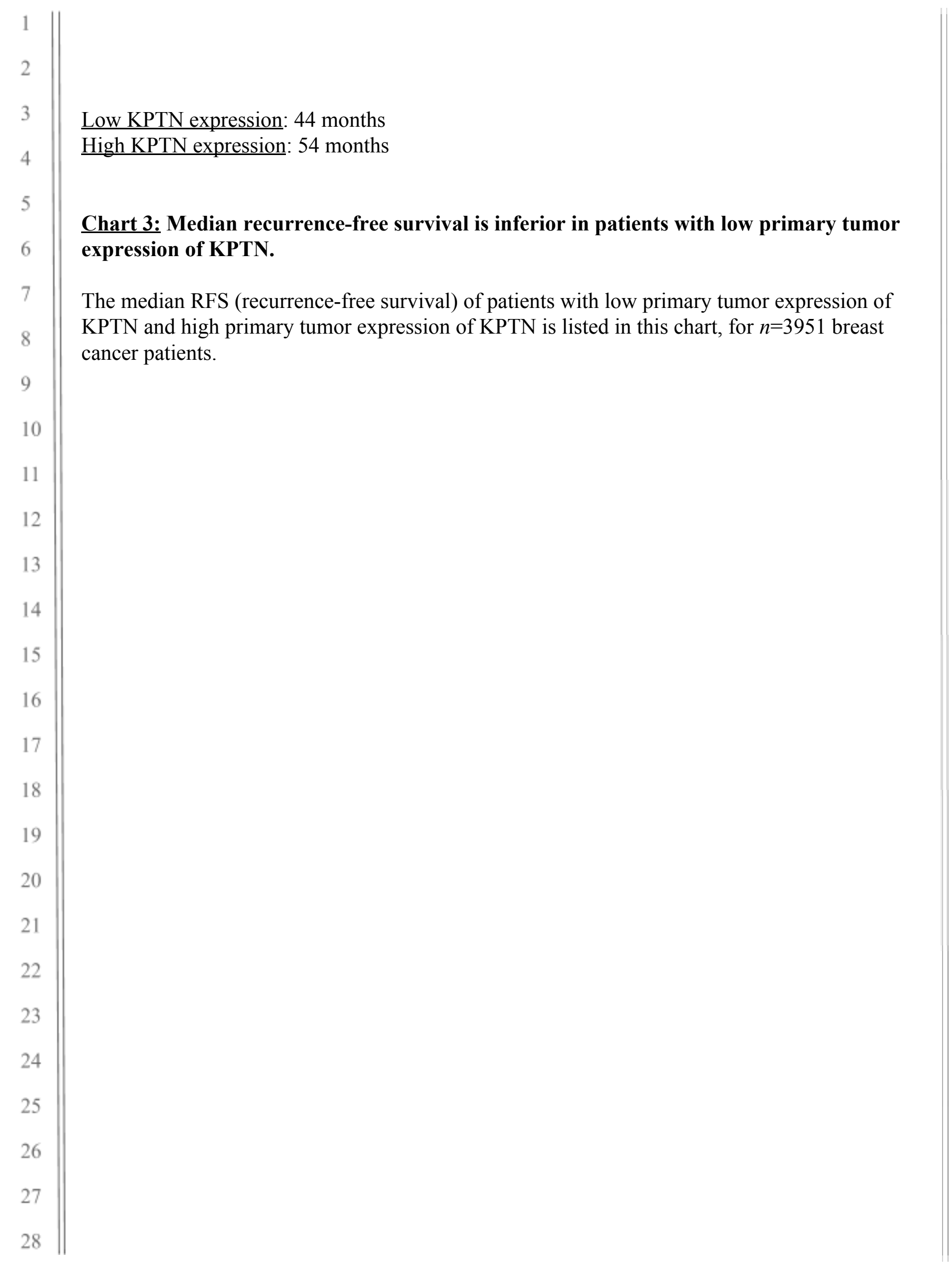

